\title{
Von der „Wirklichkeit der Strasse“ zur „Wirklichkeit der Seele“. Hermann Bahrs Beitrag zur modernen österreichischen Literatur
}

De la « réalité de la rue » à la "réalité de l'âme ». La contribution de Hermann Bahr à la littérature autrichienne de la " modernité » From the "reality of the street" to the "reality of the soul". Hermann Bahr's contribution to modern Austrian literature

\section{Giovanni Tateo}

\section{(2) OpenEdition}

\section{Journals}

Édition électronique

URL : http://journals.openedition.org/austriaca/542

DOI : 10.4000/austriaca.542

ISSN : 2729-0603

Éditeur

Presses universitaires de Rouen et du Havre

Édition imprimée

Date de publication : 1 juin 2018

Pagination : 73-88

ISBN : 979-10-240-1233-9

ISSN : 0396-4590

\section{Référence électronique}

Giovanni Tateo, „Von der „Wirklichkeit der Strasse“ zur „Wirklichkeit der Seele“. Hermann Bahrs Beitrag zur modernen österreichischen Literatur", Austriaca [Online], 86 | 2018, Online erschienen am: 01 Juli 2020, abgerufen am 28 Januar 2021. URL: http://journals.openedition.org/austriaca/542 ; DOI: https:// doi.org/10.4000/austriaca.542 


\title{
Von der „Wirklichkeit der Strasse“ zur „Wirklichkeit der Seele“
}

\author{
HERMANN BAHRS BEITRAG ZUR MODERNEN \\ ÖSTERREICHISCHEN LITERATUR
}

In der breit angelegten Debatte über die „Moderne“, die in Österreich am Ende des 19. Jahrhunderts angestoßen wurde, gebührt zweifelsfrei dem schaffensfreudigen Romancier, Dramaturgen und pointiertfeinsinnigen Literaturkritiker Hermann Bahr eine Schlüsselposition. Der politisch stark engagierte Wiener Student entdeckte seine künstlerische Berufung während seiner Aufenthalte in den beiden europäischen Metropolen Berlin und Paris. So erinnert er sich in seiner Autobiographie:

„Bin am Leben, aber lebe nicht“, schreibt Tolstoi trostlos einmal in sein Tagebuch. Alle meine wilden, dummen, schändlichen Streiche waren nur Verirrungen der quälenden Sehnsucht, endlich einmal nicht bloß am Leben zu sein, sondern zu leben. Erst die drei Berliner Jahre gaben mir die Kraft dazu. Berlin verdank ich's, daß ich reif wurde für Paris, das mich entschied: für die Kunst. ${ }^{1}$

Insbesondere in Berlin, wo sich der junge Bahr bei seiner Einschreibung in den Studiengang der Nationalökonomie an der Friedrich-WilhelmsUniversität im Mai 1884 vom Mythos der Großstadt als Zentrum der erhofften Epochenwende angezogen fühlte, nahmen seine Ideen zunehmend konkretere Formen an. Die Hauptstadt des jungen deutschen Kaiserreichs bot in jenen Jahren das Bild einer modernen, pulsierenden Metropole, die ihren ehemals provinziellen Anstrich abgestreift hatte und sich ebenso durch die Vielfalt kultureller Phänomene wie die Verdichtung scharfer sozialer Gegensätze kennzeichnete. Bahrs persönliche Berührung mit Berlin stand unter dem Vorzeichen einer enthusiastischen Haltung, die rasch einer Enttäuschung weichen sollte; denn sein Enthusiasmus fußte auf einer eher oberflächlichen Kenntnis der

1. Hermann Bahr, Selbstbildnis, Berlin, S. Fischer, 1923, S. 166. 
preußischen Realität sowie einer Geisteshaltung des Bohemien, die beispielsweise mit der Begeisterung für Bismarck eindrucksvoll die eigene Wienerische „Provinzialität“ verriet. Auf genau diese Erfahrung scheint Bahr in einer Passage seiner Studie Die neue Psychologie von 1890 rückblickend mit offenkundiger Ironie anzuspielen:

Irgend ein Fall. Einen jungen Menschen, Wiener z.B., aus der Rasse von 1860, also mit zwanzig Jahren, sehr „national“, Bismarckverschwärmt und „PreuBenseuchler“, wie damals das offizielle Beiwort hieß. Trotzdem natürlich - ohne es zu merken - eingewienert durch und durch [...]. Der also von irgend einem Zufall an die Spree verworfen. Hört, sieht, denkt. Wie er wiederkommt, nach einem Jahr, ists aus - weg mit dem schwarz-weißen Enthusiasmus, spurlos verschwunden, und mit einem ganz Donauwalzerisch angewandelt. $^{2}$

Die „Leidenschaft für das Wienerische“ hingegen speiste sich aus der Lektüre europäischer Literatur. Bahr las zunächst Zola, den er als „Roßkur" gegen die Lektüre der stehen gebliebenen Epigonen im Stile Paul Heyses definiert; ${ }^{3}$ auf die Entdeckung des französischen Autors folgte rasch die des Theaters von Henrik Ibsen. Im Mai 1887 wohnte Bahr der Berliner Inszenierung von Rosmersholm bei, mit Charlotte Frohn und Emanuel Reicher in den Hauptrollen; er betont die hohe Bedeutung dieser Aufführungen, die ihn dazu bewegt hatten, erstmals ein Berliner Theater zu betreten. ${ }^{4}$

Bahrs „Bekehrung“ zur Literatur steht also im Zeichen der während seiner Berliner Zeit reifenden Reflektionen. Es sind die Jahre der Begründung des deutschen Naturalismus, dessen Programm sich 1885 festigte bzw. in seinen poetologisch-kreativen Forderungen zuspitzte und der damit als literarische Bewegung in seine zentrale Phase eintrat. Zwischen den späten 7oer Jahren und der Mitte der 8oer Jahre veröffentlichten die Autoren des Realismus ihre bedeutendsten Werke, in denen sich zwar ein spürbar komplexes und zugleich höchst problematisches Verhältnisses zur historischen Realität Bahn brach, zugleich aber die literarische

2. Hermann Bahr, Die Überwindung des Naturalismus, Dresden und Leipzig, E. Pierson's Verlag, 1891, S. 113.

3. Hermann Bahr, Selbstbildnis, op. cit., S. 191-192.

4. Vgl. Heinz Kindermann, Hermann Bahr. Ein Leben für das europäische Theater, Graz-Köln, Verlag Hermann Böhlaus Nachf., 1954, S. 29. Die Entdeckung Ibsens datiert schon in die erste Wiener Phase, fällt also bezeichnender Weise zeitlich mit Bahrs Wechsel von den humanistischen zu den politisch-ökonomischen Studien zusammen; diese standen zweifellos in größerem Einklang mit den ErneuerungBestrebungen, die den künftigen Theoretiker der „Moderne“ damals beschäftigten. 
Tradition des 19. Jahrhunderts noch nicht grundsätzlich in Frage gestellt wurde. Erst die neue, von einem nunmehr geschärften literarischen Bewusstsein geleitete Schriftsteller-Generation, verspürte das Bedürfnis, den eigenen Erkenntnishorizonts zu erweitern und sich so bewusst den Problemen zu stellen, denen der Romanautor des 19. Jahrhunderts ausgewichen war, indem er die Realität poetisch verklärte.

Im Frühjahr 1882, zeitgleich zur Gründung der von Michael Georg Conrad geleiteten Naturalisten-Gruppe in München, erschien die erste Ausgabe der Zeitschrift Kritische Waffengänge. Darin führten die Brüder Heinrich und Julius Hart mit zunehmender Entschiedenheit ihren literarischen Diskurs fort, den sie in den Jahren 1877 bis 1879 mit den Zeitschriften Deutsche Dichtung sowie Deutsche Monatsblätter, Zentralorgan für das literarische Leben der Gegenwart begonnen hatten, die sozusagen als Geburtszeugnis des Berliner Naturalismus betrachtet werden können. Bahr machte die persönliche Bekanntschaft von Arno Holz, der 1885 seine Gedichtsammlung Buch der Zeit mit dem programmatischen Untertitel Lieder eines Modernen veröffentlicht hatte. Er verbrachte mit ihm seine Abende auf dem Balkon des Café Bauer und besuchte ihn 1887 in Niederschönhausen, wohin Holz sich zusammen mit Johannes Schlaf zurückgezogen hatte, um zu meditieren und mit den im Umkreis des Vereins „Durch!“ entwickelten neuen Theorien zu experimentieren.

Nach Ableistung seines Militärdiensts 1887/1888 fasste Bahr den Entschluss nach Paris zu reisen. Dort kam er sowohl direkt in Berührung mit dem französischen Naturalismus - als dem Ursprungsmodell des deutschen Naturalismus, das dieser neu interpretiert hatte-, als auch mit den gegenläufigen Tendenzen zum Naturalismus. Schon vorher scheint Bahr allerdings auf der Suche nach einer Literatur der Zukunft gewesen zu sein, die eine nur durch die „Überwindung“ des Naturalismus zu verwirklichende Synthese erforderte; eine solche Synthese verkündete er bereits 1887, also noch vor der Gründung der Berliner „Freien Bühne“ und der Entstehung von Gerhart Hauptmanns Drama Vor Sonnenaufgang (1889). ${ }^{5}$

Der Pariser Aufenthalt vom Herbst 1888 bis zum Frühjahr 1890 bot Bahr deshalb die Gelegenheit, die während der Berliner Jahre gereiften Überlegungen zu präzisieren und auszuarbeiten. Seinen wichtigsten Niederschlag fanden dieser Reflexionsprozess in dem 1890 publizierten Band Zur Kritik der Moderne, der mehrere zwischen 1886 und 1889

5. Donald G. Daviau, Der Mann von Übermorgen. Hermann Bahr 1863-1934, Wien, Böhlau, 1984, S. 70-85. 
zunächst einzeln erschienene Beiträge Bahrs zusammenführt. Seine Berührung mit den jüngsten Erfahrungen der französischen Literatur, mit Baudelaire, Gautier, Huysmans und Barrès, bestätigte Bahr in seiner Überzeugung von der Notwendigkeit einer Erneuerung, die zwangsläufig die Überwindung der positivistischen und pseudowissenschaftlichen Konzepte der naturalistischen Ästhetik einschloss. ${ }^{6}$

In Paris, wo Bahr, wie er später in seiner „Autobiographischen Skizze“ festhält, in seinem Stil und seiner Haltung des „Französelns“ bezichtigt wurde, ${ }^{7}$ festigte sich nicht nur die Homogenität seines Stils als Kritiker, sondern Bahr fand vor allem auch tragfähigere kulturelle Bezugsmodelle für das methodologische Gerüst des eigenen Diskurses, der stets den unmittelbaren persönlichen Eindruck bevorzugt. ${ }^{8}$ So verortet er sich selbst nunmehr eindeutig in einer intellektuellen Tradition, die jenseits des literarischen mainstream verläuft und von Sainte-Beuve, Taine, Paul Bourget und Anatole France bis zu Jules Lemaître und Maurice Barrès reicht. 1884 war Huysmans' Roman À rebours erschienen, der, wie Bahr an einer Stelle präsent macht, rasch „ein Brevier der suchenden Jugend geworden " war. ${ }^{9}$

6. Der Notwendigkeit einer “Überwindung” hatte Bahr bereits in seiner Schrift „Henrik Ibsen" von 1887 Ausdruck verliehen, die in der Wiener Zeitschrift Deutsche Worte, Jg. VII, Heft 8/9, August und September 1887, S. 338-353, erschien und später in die kritische Sammlung Zur Kritik der Moderne, Zürich, Verlags-Magazin, 189o, S. 59-79, aufgenommen wurde. Vgl. Giovanni Tateo, „Heute mittag bricht für die deutsche Literatur eine neue Epoche an.' Henrik Ibsens Gespenster im Urteil Theodor Fontanes, Gerhart Hauptmanns und Hermann Bahrs“, in Fontane Blätter, XC, 2010, S. 86-109, sowie Lukas Mayerhofer, „Facetten einer Rezeption: Hermann Bahr und Henrik Ibsen“, in Johann Lachinger et al. (Hg.), Hermann Bahr - Mittler der europäischen Moderne, Linz, Adalbert-Stifter-Institut des Landes Oberösterreich, 2001, S. 71-86.

7. In der anlässlich seines 50. Geburtstags in der Zeitschrift Illustriertes Wiener Extrablatt (Jg. 42, Nr. 196, 1913, S. 6) publizierten „Autobiographischen Skizze“, schreibt Bahr die ihm wiederholt vorgehaltene eigene „Französisierung“ der barocken Atmosphäre zu, die er in seiner Heimatstadt Salzburg eingeatmet hatte. Vgl. Das HermannBahr-Buch, S. Fischer, Berlin 1913, S. 15-18, hier S. 16. In der Demolirten Literatur spricht Karl Kraus mit bissig-ironischem Ton von der „französierende[n] Art des Meisters" (Wiener Rundschau, Bd. 1, Nr. 1, 15. November 1896, S. 19-27: hier S. 22).

8. Zu Bahrs eigentümlichen essayistischen Verfahren, das jede an ästhetischen Regeln und Dogmen orientierte Kritik ablehnt und als „impressionistisch“ bezeichnet werden kann, vgl. Hartmut Steinecke, „,Verwandlungskünstler'? Zur Literaturkritik des Jungen Wien“, in Benjmin Bennett et al. (Hg.), Probleme der Moderne. Studien zur deutschen Literatur von Nietzsche bis Brecht. Festschrift für Walter Sokel, Tübingen, Max Niemeyer Verlag, 1983, S. 101-116.

9. Hermann Bahr, Selbstbildnis, op. cit., S. 237. Einige Seiten zuvor wird der Roman allerdings auch als „ein Handbuch des Dilettantismus" definiert (S. 232). 
Nach seiner Rückkehr von einer Spanien- und Nordafrikareise erreichte Bahr in Paris das von Arno Holz unterbreitete Angebot einer Zusammenarbeit an der Zeitschrift Freie Bühne für modernes Leben, die 1890 in Berlin gegründet worden war. ${ }^{10}$ Bahr nahm die Einladung an und kehrte am 1. Mai 1890 nach Berlin zurück. Die sich anschließend entwickelnde Zusammenarbeit dokumentiert die tiefe geistige Distanz zur literarischen Szene der deutschen Hauptstadt, in die Bahr während seiner zweijährigen Abwesenheit gerückt war. Denn während man hier noch ganz im Bannkreis der naturalistischen Bewegung weiterhin darum bemüht war, mit den Relikten des Epigonentums abzurechnen, sah Bahr selbst im Einklang zu den aktuellen europäischen Tendenzen den Naturalismus längst als abgeschlossen an und wies bereits darüber hinaus: „Und ich, so gierig nach auserlesenen Sensationen, so stolz auf meine Nervenkunst, fand mich unter Leuten, die Nerven überhaupt nicht kannten, ja die sich geschämt hätten und erschrocken wären, Nerven zu haben." 11

Inzwischen hatte der Schriftsteller Eduard Michael Kafka 1889 die bei E. M. Rohmer in Brünn publizierte literaturkritische Monatsschrift Moderne Dichtung ins Leben gerufen. Diese hatte es sich mit Blick auf die europäische, insbesondere aber auf die Berliner Literatur, zur besonderen Aufgabe gemacht, auch die österreichische Literaturszene $\mathrm{zu}$ erneuern, indem sie gezielt alle positiven heimischen Zeugnisse förderte, die dafür bereits zu registrieren waren. Nach einem lebhaften Briefwechsel mit dem damals noch unbekannten Kafka willigte Bahr, der zu diesem Zeitpunkt noch in Paris wohnte, in dessen Vorschlag der

10. Eine detaillierte Rekonstruktion von Bahrs journalistischer und literar(krit)ischer Tätigkeit in Berlin in den Jahren 1890-1891 liefert Gregor Streim, „,Die richtige Moderne'. Hermann Bahr und die Formierung der literarischen Moderne in Berlin“, in Hofmannsthal-Jahrbuch, 4, 1996, S. 323-359. Der Beitrag wurde in erweiterter Form, d.h. unter Einbeziehung der Phase nach Bahrs Rückkehr nach Wien, in das Kapitel „Hermann Bahr und die Differenzierung der literarischen Moderne zwischen Wien und Berlin“, aufgenommen, in Peter Sprengel, Gregor Streim, Berlin und Wiener Moderne. Vermittlungen und Abgrenzungen in Literatur, Theater, Publizistik. Mit einem Beitrag von Barbara Noth, Wien, Köln, Weimar, Böhlau 1998, S. 45-114. Bahrs Zusammenarbeit mit der Freien Bühne rekonstruiert Gerd-Hermann Susen, „,Das Alte kracht in allen Fugen!“. Hermann Bahr und die Freie Bühne für modernes Leben“, in Martin Anton Müller et al. (Hg.), Hermann Bahr: Österreichscher Kritiker europäischer Avantgarden, Bern, Peter Lang, 2014, S. 39-54.

11. Hermann Bahr, Selbstbildnis, op. cit., S. 256. 
Mitarbeit an der neuen Zeitschrift ein. Den Inhalt des Briefwechsels mit Kafka charakterisiert Bahr 1899 rückschauend in dem Artikel „Zehn Jahre“:

Es ist nicht von Naturalismus, nicht von der Decadence und nicht vom Symbolismus die Rede; nichts deutet an, dass eine Schule oder eine Partei gestiftet werden soll. [...] Jeder, der dichtet, soll dabei sein - das ist das einzige Programm. Eine Literatur - endlich eine Literatur in Oesterreich!, das ist der ewige Refrain. ${ }^{12}$

Für Kafkas Zeitschrift schrieb Bahr 1890 neben dem Leitartikel Die Moderne, der die erste Ausgabe eröffnet, eine Reihe zentraler Aufsätze, wie Zola, Die neue Psychologie, Vom Stile, die 1891 gemeinsam in den Band Die Überwindung des Naturalismus Eingang fanden. Bahrs Zusammenarbeit mit dem „eingeschworene[n] Naturalisten“ Kafka, liegt keinesfalls - wie zuweilen vermutet - ein Missverständnis zugrunde: ${ }^{13}$ genauer betrachtet hatte der traditionelle Wortschatz mit den Aufsätzen zur Überwindung des Naturalismus eine semantische Veränderung erfahren. In seiner Rezension zum ersten Aufsatzband Zur Kritik der Moderne von 1890 rechnete Kafka dessen Verfasser zwar zum „Fähnlein“ der „Sozialisten der modernen Literatur“, zusammen mit den Naturalisten Max Kretzer, Karl Henckell, Arno Holz und Gerhart Hauptmann. Zugleich hob er in seinem scheinbar positiven Urteil aber auch die Unterschiede und die Neuheit Bahrs im Verhältnis zu den „gewöhnlichen Vorstellungen des modernen Naturalismus hervor“. Von dieser Verschiedenheit kündete die der Kunst zugewiesene Aufgabe, eine „Synthese von Naturalismus und Romantik“ herzustellen. ${ }^{14}$ Kafkas Zeitschrift änderte am 1. April 1891 ihren Namen in Moderne Rundschau und verlegte kurz nach Bahrs Wiener Rückkehr ihren Redaktionssitz in die habsburgische Hauptstadt. ${ }^{15}$ Neben den literarischen Beiträgen der Naturalisten selbst (Hauptmann, Michael Georg Conrad, Arno Holz u.a.) fanden nun zunehmend auch solche junger Autoren Platz, die bereits gegenläufige oder zumindest abweichende Tendenzen zum Naturalismus vertraten: Max Dauthendey, Detlev von Liliencron und Richard Dehmel, Felix

12. Hermann Bahr, Bildung, Berlin und Leipzig, Insel-Verlage bei Schuster \& Loeffler, 1900, S. 173. Vgl. dazu auch Selbstbildnis, op. cit., S. 277-79.

13. Heinz Kindermann, Hermann Bahr, S. 49: „Kafka war ein eingeschworener Naturalist. Und er nahm Bahrs Wahrheitsfanatismus für eine eindeutig-naturalistische Programm-Erklärung“.

14. Eduard Michael Kafka, „Zur Kritik der Moderne“, Moderne Dichtung, Bd. 1, Heft 2, Nr. 2, 1. Februar 1890, S. 120-122.

15. Fritz Schlawe, Literarische Zeitschriften 1885-1910, Stuttgart, Metzler, 1965, S. 31-32. 
Salten und Felix Dörmann, Arthur Schnitzler, mit seiner Erzählung Reichtum und dem Einakter Anatols Hochzeitsmorgen, schließlich Hugo von Hofmannsthal, mit einigen seiner bedeutendsten Gedichte und der dramatischen Studie Gestern sowie einer Reihe unterschiedlicher kritischer Schriften.

Die von Bahr in jenen Jahren proklamierte Überwindung des Naturalismus setzte bei einer tiefgreifenden Analyse des literarischen Phänomens selbst an, die sowohl darauf zielte, die innersten Beweggründe seiner Poetik zu ergründen, als auch die spezifischen Voraussetzungen, die die Rezeption des französischen Naturalismus in Deutschland geleitet hatten. Bahrs Urteil fiel besonders gegenüber den Berliner Naturalisten deutlich negativ aus, die - wie er 1893 in der Studie Das jüngeste Deutschland betonte - in seinen Augen „bloss das Ausland äfften“, ohne selbst irgendeinen kreativen Beitrag geleistet zu haben: „sie haben nur aus der Weltliteratur genommen, nichts in die Weltliteratur gegeben“. ${ }^{16}$ Im französischen Naturalismus sah Bahr „eine nothwendige und unvermeidliche Reaction gegen die phantastischen Ausschweifungen der entarteten Romantik“, ${ }^{17}$ der die ,jüngsten Deutschen“ ihrerseits das Konzept der Wahrheit entgegengesetzten, das sich allerdings rasch in das „neue Dogma der Kunst“ gewandelt hatte. Dieses duldete nur eine bestimmte Form der Wahrheit, nämlich „die ganze Alltäglichkeit um uns, ohne Dazwischenkunft des Künstlers, das Leben da draußen und nichts als das äußere Leben, so wie sie ist", ${ }^{18}$ ohne ein Gespür dafür $\mathrm{zu}$ entwickeln, dass neben der äußeren auch eine innere Wahrheit existierte, welche die "Wirklichkeit der Seele " umschloss. ${ }^{19}$ Entsprechend hatten sich die französischen Naturalisten für Bahr von den deutschen dadurch abgehoben, dass sie die äußere Wahrheit nicht als eine allgemein verbindliche betrachteten:

Sie sammelten wohl genaue, umständliche und grausame Dokumente des Lebens, aber nur um in ihnen und durch sie sich selber auszudrücken, die eigenen Gedanken, die eigenen Entrüstungen, die eigenen Wünsche, immer nur die letzte Natur des Dichters selbst: der Naturalismus war ihnen ein Mittel der Form, dahinter blieb eine nur besser verkappte, wirksam maskierte Romantik. ${ }^{20}$

16. Hermann Bahr, Studien zur Kritik der Moderne, Frankfurt a. M., Rütten \& Loening, 1894, S. 68-69.

17. Ebd., S. 61.

18. Ebd.

19. Ebd. Hermann Bahr, Zur Kritik der Moderne, S. 223.

20. Hermann Bahr, Studien zur Kritik der Moderne, op. cit., S. 61-62. 
Die Differenzen zwischen dem französischen Naturalismus und dessen formalistisch erstarrter deutscher Variante, die das kreative Moment zunehmend in programmatischer Weise zu begründen versuchte, markierte Bahr in einem scharfsinnigen Artikel vom September 1890 mit der Überschrift „Naturalismus und Naturalismus“. Darin analysierte er zwei Theaterstücke aus dem Jahre 1890, die in einen spiegelbildlichen Bezug zueinander gerückt sind: Chapons von Lucien Descaves und Georges Darien und Die Familie Selike von Arno Holz und Johannes Schlaf. Bahr zeigt hier, dass der Realitätsanspruch von den französischen und deutschen Naturalisten auf jeweils unterschiedliche Weise angestrebt wird. Geht es den französischen Naturalisten darum, „den Künstler in der lebendigen Wirklichkeit erst recht zu bestätigen und zu bewähren“, so verzichtet der deutsche Naturalist auf die Figur des Künstlers, „um ihn in der wirklichen Fülle zu ersticken“. Für die Franzosen sind „alle Bemühung um die Wirklichkeit nur Mittel im Dienst des künstlerischen Temperaments“, für die Deutschen hingegen ist „die Wirklichkeit der letzte Zweck, dem alles Temperament des Künstlers dient“". ${ }^{21}$

Bahr kommt also das Verdienst zu, die der naturalistischen Poetik inhärenten Widersprüche klar erkannt zu haben, indem er die immanent wirksamen Kräfte aufdeckte, die sich deren theoretischen Vorsätzen erkennbar widersetzten; darüber hinaus wies er aber auch als Erster darauf hin, dass der Naturalismus bei seinem „konsequent[en]“ Streben nach einer absoluten Nachahmung von Haus aus immer auch impressionistisch war.

In seinem Artikel „Wahrheit! Wahrheit!“ entfaltete Bahr eine vollständige Analyse der philosophischen Unzulänglichkeit der naturalistischen Ästhetik, die "langsam und elend verstorben ist“, 22 steht sie doch mit ihrer Unterscheidung zwischen dem „Ding an sich“ und seiner „Erscheinung“" noch eindeutig in der Tradition von Kants Erkenntnistheorie. ${ }^{23}$ Der Naturalismus hatte die Illusion gehegt, die Phänomene der Wirklichkeit objektiv erfassen zu können, ohne zu der Einsicht gelangt zu sein, dass die Welt nur als subjektive Erscheinung ausgedrückt werden kann; denn, wie Bahr in einem Beitrag über „Die Sezessionisten in Wien“ bemerkt, „[ist] es dem Menschen versagt [...], die Dinge zu

21. Hermann Bahr, Die Überwindung des Naturalismus, op. cit., S. 54-55.

22. Ebd., S. 145.

23. Manfred Diersch, Empiriokritizismus und Impressionismus, Berlin, Rütten \& Loening, 1977, S. 62. 
fassen, wie sie sind, und [..] er [muss] sich bescheiden [...] sie zu nehmen, wie sie ihm scheinen". ${ }^{24}$

Unter diesen Vorzeichen erscheint die von Bahr gestellte Frage „Was ist denn überhaupt Wahrheit “25 quasi als wörtliche Übersetzung von Pontius Pilatus' Ausspruch „quid est veritas“, der ja die Bestürzung einer ganzen Kultur ausdrückte bzw. die Komplexität einer eher philosophischen als im eigentlichen Sinne religiösen Haltung der heidnischen Aristokratie gegenüber dem rigorosen Anspruch auf eine einzige gültige Wahrheit spiegelte. Bahr begreift Wahrheit stets als eine Manipulation des Bewusstseins, „eine zusammengesetzte Wirkung“, insofern sie „,von ihrer äußeren Ursache und von ihrer inneren Form“ bestimmt ist, „weil jeder Mensch aus anderen Sinnen und anderen Nerven ein anders, sein besonderes Bewußtsein, jeder Mensch auch eine andere, seine besondere Wahrheit hat" ${ }^{26}$ Eine solche Sichtweise bereitete den Boden für die Psychologen, die ebenfalls auf der Suche nach Wahrheit waren, allerdings einer Wahrheit, die im Inneren des Menschen zu finden war. Der von Bahr eingeschlagene Weg sollte ihn selbst direkt zum Empiriokritizismus Ernst Machs hinführen, wenn auch erst vierzehn Jahr später, im Zuge von dessen Wiederentdeckung durch den Großteil der Schriftsteller des Jungen Wien. Gegen die „Kompromiß- und Majoritätswahrheit" redet Bahr also einer Wahrheit das Wort, die ,in der Menschenseele drin, auf den wirren Nerven, unter den bangen Geheimissen der Sinne" zu suchen ist. ${ }^{27}$ Ähnlich wie für Mach existiert die Wirklichkeit ausschließlich als Erfahrung des Ich; ein solches Ich erweist sich allerdings zugleich als janusköpfig, insofern es einerseits Katalysator des Realen ist, andererseits aber auch „Konstruktion, willkürliche Anordnung, Umdeutung und Zurichtung der Wahrheit, die jeden Augenblick anders gerät, wie es einem gerade gefällt, eben nach der Willkür der jeweiligen Stimmung “. ${ }^{28}$

In den neunziger Jahren repräsentierte die Erfahrung der „Freien Bühne" in Berlin sowohl das Moment des reifen Naturalismus, der die Phase der theoretischen Manifeste hinter sich gelassen und die Notwendigkeit der Unterstützung und Förderung der eigenen Kunst erkannt hatte, als auch die sich schärfende Wahrnehmung neuer literarischer

24. Hermann Bahr, Renaissance, Berlin, S. Fischer, 1897, S. 194.

25. Hermann Bahr, Die Überwindung des Naturalismus, op. cit., S. 143.

26. Ebd., S. 144.

27. Ebd., S. 146.

28. Ebd., S. 149. 
Erscheinungsformen, die sich neben dem Naturalismus sowie immer häufiger auch in seinem Namen herausbildeten.

Diese Prämissen liefern den Ausgangspunkt für Bahrs Studie Die Überwindung des Naturalismus, die dem 1891 publizierten Band seinen Titel gibt und sich gewissermaßen als Leitmotiv durch die Argumentation der darin vereinigten Beiträge zieht, wie bereits an dem sinnfälligen Motto aus Pauls Bourget Etudes et portraits abzulesen ist. ${ }^{29}$ Bahr bekräftigt zunächst die Bedeutung des Naturalismus als bloße Episode, als historisch bedingtes „Intermezzo“ zwischen Altem und Neuem und konstatiert das Fehlen schlüssiger alternativer Lösungen, die auch er selbst ausdrücklich nicht besitzt. Im Weiteren konzentriert er sich darauf, minuziös nach sämtlichen Spuren zu suchen, die darauf hinweisen, dass die gegenwärtige Kunst sich selbst dort, wo sie ihren Ausgangspunkt im Naturalismus findet, in die entgegengesetzte Richtung bewegt. Als Paradebeispiel dient Bahr die Psychologie. Diese treibt die im „konsequenten Naturalismus" bereits enthaltenen Elemente bis ins Extrem und provoziert so eine Krise der naturalistischen Ästhetik, die nämlich geradezu auf den Kopf gestellt wird: der Künstler fungiert nicht länger als ein Instrument der Wirklichkeit, sondern vielmehr liefert umgekehrt die Wirklichkeit das Material, aus dem der Künstler schöpfen muss, um die eigene Natur auszudrücken. Die Kunst hat ihren Ursprung somit im Menschen selbst, der in seiner nervlichen Verfasstheit erfasst wird, in der plötzlichen Wandlung der Gemütszustände und den sich wirr anhäufenden gedanklichen Assoziationen. Als Überwindung der klassischen Vernunft wie auch des romantischen Begriffspaars von Sinnen und Leidenschaften weist die Nervenkunst somit einen möglichen Ausweg aus den Aporien der Gegenwart. Dabei unterscheidet Bahr in seiner Analyse des Naturalismus drei Phasen: das Interesse an der Außenwelt; das Interesse an der Innenwelt; das Interesse an der Sphäre des Kontakts zwischen Außen- und Innenwelt, die als das „Nervöse“ definiert wird, im Sinne von dynamischer Wahrheit, Veränderung und Mobilität. Wie Bahr später, im Zuge seiner genaueren Ausarbeitung des Konzepts der Décadence, darlegte, suchte diese Bewegung in den Nerven ihr eigenes Ich zu fassen, ähnlich wie vor ihr die Klassik im Geiste, die Romantik

29. Das Motto: „La vie dans l'Esprit, comme dans la Nature, échappe à la définition. Elle est chose sacrée et qui ne relève que de la Cause Inconnue" wird von den beiden letzten Sätzen einer langen Fußnote zum Kapitel „Réflexions sur l'art du roman“ wieder aufgenommen, vgl. Paul Bourget, Études et portraits, vol. I, Paris, Alphonse Lemerre, 1889, S. 260-279, hier S. 277. 
im Gefühl und der Naturalismus in der ihn umgebenden Außenwelt. ${ }^{30}$ Zunächst einmal beabsichtigte Bahr aber mit seinem insistenten Bezug auf die „Nerven“ die Präsenz einer Art von „Mechanismus“ aufzuzeigen, der angesichts seiner hohen, unmittelbar wirksamen Reaktionsfähigkeit die notwendige Empfänglichkeit des Künstlers gegenüber den äußeren Reizeinwirkungen sicherstellte. ${ }^{31}$ Bahr erkennt die Signale dieser Veränderung in der Malerei, in der Musik wie in der Literatur, konkret in Pierre Puvis de Chavanne, Edgar Degas, Georges Bizet und Maurice Maeterlinck.

Von hier aus erschließt sich auch die Perspektive Eduard Michael Kafkas in seiner Rezension der gesammelten Ausgabe von Bahrs Aufsätzen, die unter dem Titel Die Überwindung des Naturalismus erschienen war. Denn Kafka sieht hier im „neuesten Bahr“ nicht so sehr den "Grabredner des Naturalismus“, als vielmehr eine „Phänomenologie des Naturalismus", die den Übergang der literarischen Bewegung von der „Wirklichkeit der Straße“, über die „Wirklichkeit der Seele“ hin zur „Wirklichkeit auf den Nerven“ Revue passieren ließ, indem sie „ein treuliches Bild" von diesem Übergang erstellte. ${ }^{32}$

Im August/September 1890, fast gleichzeitig mit Bourgets Schrift über den französischen Naturalismus, druckte die Zeitschrift Moderne Dichtung in zwei Teilen Bahrs Aufsatz „Die neue Psychologie“ ab. Darin sprach sich Bahr nunmehr ganz entschieden für eine neue Psychologie aus, wie er sie bereits in der Krisis des Naturalismus gefordert hatte, d.h. „eine Psychologie, welche der langen Gewohnheit des Naturalismus Rechnung trägt" ${ }^{33}$ Die Rückgewinnung einer solchen Psychologie dient dabei einerseits der Entfernung vom Naturalismus, dem Übergang von den choses zu den âmes, ohne dass andererseits jedoch das methodologische Erbe des Naturalismus auszuschlagen ist, nämlich die Untersuchung der états. Die naturalistische Verfahrensweise wird auf

30. Hermann Bahr, Studien zur Kritik der Moderne, op. cit., S. 72.

31. In Bezug auf den von Bahr 1891 publizierten dramatischen Dreiakter Die Mutter definierte Hofmannsthal den Autor in Analogie zu den Figuren seines Stücks als „Künstler des gesteigerten Lebens, der raffinierten Empfindung, der potenzierten Sensation“. Hugo von Hofmannsthal, Sämtliche Werke. Kritische Ausgabe, hg. von Rudolf Hirsch et al., Bd. XXXII: Reden und Aufsätze. 1, hg. von Hans-Georg Dewitz, Olivia Varwig, Mathias Mayer, Ursula Renner und Johannes Barth, Frankfurt/M., S. Fischer, 2015, S. 13-17, hier S. 14.

32. Eduard Michael Kafka, „Der neueste Bahr“, Moderne Rundschau, Bd. 3, Heft 5/6, 15. Juni 1891, S. 220-222, hier S. 221.

33. Hermann Bahr, Die Überwindung des Naturalismus, op. cit., S. 67. 
diese Weise neu interpretiert und mit der Psychologie auf ein von den Naturalisten selbst nicht erfasstes Untersuchungsfeld umgelenkt. Entsprechend beabsichtigt Bahr "die deterministische Methode“ nicht mehr ausschließlich bzw. nicht mehr in erster Linie auf das milieu anzuwenden, sondern auf die "Gefühle“. Dabei spürt er deren inneren Bezügen nach, indem er die Verknüpfungsweisen der Gefühle mit ihren Ursprüngen und äußeren Situationen bestimmt, ohne allerdings, wie in der Psychologie des „alten“ Romans, den Menschen in seinem äußeren Kontext aus dem Blick zu verlieren. ${ }^{34}$ Dieselbe naturalistische Methode zur Objektivierung der äußeren Zustände steht nun im Dienst der „inneren Seelenstände“. ${ }^{35}$

Die Jahre der parallel verlaufenden Mitarbeit an Brahms Freie Bühne und E. M. Kafkas Moderne Dichtung, zwischen dem Ende der Pariser Zeit und dem zweiten kurzen Berlin-Aufenthalt, stehen also für die fortschreitende Präzisierung des Konzepts der Moderne und ebneten damit zugleich den Weg für Bahrs Rückkehr nach Wien. Diese war nicht geplant, sondern resultierte aus einer Reihe mehr oder weniger zufällig zusammenwirkender Umstände. Eine entscheidende Bedeutung kommt dabei dem sich festigenden Bewusstsein Bahrs für das unwiderrufliche Ende der eigenen Berliner Erfahrungen zu. ${ }^{36}$

Insbesondere in der Begegnung mit dem jungen Hofmannsthal in Wien im Herbst 1891 sah er das Signal für den Beginn einer neuen Phase, die nicht mehr ausschließlich durch die theoretische Reflexion geprägt war, sondern ebenso durch die verstärkte Bemühung um die Förderung der neuen Literatur. ${ }^{37}$ Nachdem Bahr sich rasch in die Wiener literarischen Kreise eingeführt hatte, widmete er sich ab 1892 vorrangig einer intensiven publizistischen Tätigkeit, wobei sich sein Name in den angesehensten örtlichen Zeitschriften an exponierter Stelle findet, daneben aber auch mit Beharrlichkeit der Abfassung von Romanen und Theaterstücken. Von 1892 bis 1894 war er als Theaterkritiker für die von Emil Auspitzer geleitete Deutsche Zeitung tätig, von 1894 bis 1899 leitete er zusammen mit Heinrich Kanner und

34. Ebd., S. 105-106.

35. Ebd., S. 111.

36. Vgl. dazu Jens Rieckmann, Aufbruch in die Moderne. Die Anfänge des Jungen Wien. Österreichische Literatur und Kritik im Fin de Siècle, 2. durchges. Aufl., Frankfurt /M., Athenäum, 1986, S. 39-41.

37. Gregor Streim zufolge entwickelte Bahr die Vorstellung von der Existenz einer eigenständigen modernen Literatur in Österreich erst nach seiner Übersiedlung nach Wien; vgl. Peter Sprengel, Gregor Streim, Berliner und Wiener Moderne, S. 83. 
Isidor Singer das Wochenmagazin Die Zeit, bevor er schließlich 1899, nach seinem Bruch mit den beiden Journalisten, Theaterkritiker des Neuen Wiener Tageblatts wurde.

Hatte bis dahin die Definition der Moderne auf den Trümmern des Naturalismus im Fokus von Bahrs kritischen Bemühungen gestanden, so gewinnt in Wien zunehmend der Entwurf einer Literatur mit spezifisch österreichischen Wesenszügen an Gewicht. Für eine solche Literatur verweist er auf die von jeher bestehende kulturelle Affinität zwischen Wien und Paris und beruft sich dabei auf den „romanischen “Wesenszug der habsburgischen Tradition, den er konkret an dem für die österreichische Baukunst dominanten Stilmoment von Barock und Rokoko festmacht.

Das Bedürfnis nach Erneuerung deckte sich so quasi mit einer geographischen und kulturellen Verlagerung von Berlin nach Wien, die den „Umweg“ über Paris einschloss. Die jüngere, an erster Stelle von Marie von Ebner-Eschenbach und Ferdinand von Saar repräsentierte österreichische Literatur diente Bahr als Ausgangspunkt, um in programmatischer Weise die Unterschiede zwischen dem Berliner Naturalismus der 8oer Jahre und dessen Wiener „Überwindung“ der 9oer Jahre herauszustellen. ${ }^{38}$ So veröffentlichte Bahr 1893 in der Deutschen Zeitung zwei thematisch verwandte und zugleich komplementäre Aufsätze unter dem Titel „Das jüngste Deutschland“ und „Das junge Oesterreich“, 39 die später in den Band Studien zur Kritik der Moderne aufgenommen wurden; in diesen Aufsätzen stellte er ein grundsätzlich verschiedenes Verhältnis der Berliner und Wiener Autoren zur Generation ihrer jeweiligen Vorgänger fest. Die feindliche Haltung der ,jüngsten“ Berliner Autoren gegenüber dem Realismus des 19. Jahrhunderts, den sie allerdings nicht mit der letzten Generation der Deutschen Theodor Storm, Theodor Fontane und Wilhelm Raabe, geschweige denn mit den Schweizern Gottfried Keller und Conrad Ferdinand Meyer, sondern ausschließlich mit den als Epigonen angesehenen Autoren Paul Heyse und Friedrich Spielhagen gleichsetzten, kontrastiert für Bahr mit der „herzlichsten Verehrung“, „innigsten Liebe“ und „zärtlichsten Treue“

38. Zum Aspekt der Regionalisierung der Moderne als tragendem Moment von Bahrs publizistischer Strategie vgl. Gregor Streim, ebd., S. 91-98.

39. Vgl. dazu Giovanni Tateo, „In der Werkstatt der Wiener Moderne. Der Beitrag Hermann Bahrs", in Mauro Ponzi (Hg.), Klassische Moderne. Ein Paradigma des 20. Jahrhundert, Würzburg, Königshausen \& Neumann, 2010, S. 133-149, hier S. 144-145. 
der „jungen“ Österreicher für ihre direkten literarischen Vorgänger wie die Ebner-Eschenbach und Saar. ${ }^{40}$ Eine solche Literatur, die schon im Kontakt mit der nervösen Geisteshaltung der Zeit stand und sich in den Stillagen einer dämmrigen Weichheit entfaltete, bot sich Bahr in kongenialer Weise als Ausgangspunkt für die Neubestimmung einer österreichischen Moderne an. ${ }^{41}$

Was Bahr unter der Restituierung des „Oesterreichischen“ verstand, wird in einer so betitelten Rezension anschaulich, die er 1897 zum ersten Band des von den beiden Germanisten Johann Willibald Nagl und Jakob Zeitler herausgegebenen Deutsch-Oesterreichische Literaturgeschichte. Ein Handbuch zur Geschichte der deutschen Dichtung in Oesterreich und Ungarn verfasste. Den Ausgangspunkt seiner Überlegungen bildet eine radikale Kritik des bereits im Titel signalisierten ideologischen Ansatzes der Darstellung:

Aber wir [Österreicher] haben auch andere [Autoren], die, wenn auch mit deutschen Worten redend, sich doch keineswegs als Deutsche fühlen, indem sie andere Nerven, andere Sinne und einen ganz anderen Geist haben als die Deutschen - diese bilden unsere österreichische Literatur. Warum das Beiwort „deutsch“? Weil sie zufällig deutsch schreiben? Wird man Maeterlinck in die französische Literatur stellen, weil er zufällig französisch schreibt? ${ }^{42}$

Der Bezug auf das Werk des belgischen Autors ist symptomatisch für das gewagte, wenn nicht gar prekäre theoretische Moment, auf dem Bahr sein Unterfangen gründet. Denn seine Konstruktion einer nationalen österreichischen Identität, die er in den europäischen Kontext eingebettet und zugleich aus ihrer Verbindung mit der deutschen Kultur

40. Hermann Bahr, Studien zur Kritik der Moderne, op. cit., S. 75. Zur Gegenüberstellung der beiden österreichischen Schriftstellergenerationen vgl. Giovanni Tateo, „Ganz anders die jungen Wiener.' Hermann Bahr e il recente passato letterario austriaco come spazio di transizione al Moderno", Cultura Tedesca (Letterature del Danubio), n. 40, gennaio-giugno 2011, S. 93-104.

41. Die Debatte über das Konzept der Moderne erschöpft sich offenkundig nicht in der an sich bereits problematischen Gegenüberstellung Wien/Berlin. Den beiden Hauptstädten sind entsprechend andere Zentren hinzufügen, allen voran München, Prag und Zürich; vgl. dazu Gotthart Wunberg, „Wien und Berlin. Zum Thema Tradition und Moderne“, in: Maurice Godé et al. (Hg.), Wien - Berlin. Deux sites de la modernité - Zwei Metropolen der Moderne (1900-1930). Actes du colloque international de Montpellier (2-4 avril, 1992), Cahiers d'études germaniques. Revue semestrielle, 24, 1993, S. 219-229, jetzt in G. Wunberg, Jahrhundertwende, Tübingen, Gunter Narr, 2001, S. 176-186, hier S. 176.

42. Hermann Bahr, Bildung, op. cit., S. 113-114. 
gelöst sieht, reduziert sich auf zwei Kern-Hypothesen. Zum einen die paradox anmutende Relativierung der identitätsstiftenden Funktion der (deutschen) Sprache und zum andern die Akzentuierung zweier unterschiedlicher Herangehensweisen an die Moderne: die des (deutschen) Naturalismus und die seiner (österreichischen) Überwindung unter dem Vorzeichen des Impressionismus und Symbolismus.

Das innovative Moment von Bahrs Diskurs verdeutlicht eine Rezension seiner Überwindung des Naturalismus, die Marie Herzfeld im August 1891, unmittelbar nach Erscheinen des Bandes, in der Wiener Literatur-Zeitung veröffentlichte. Nachdem sie zunächst die Eigenständigkeit des von Bahr präsentierten kritischen Ansatzes lobend hervorgehoben hat, äußert die Verfasserin ihre Bedenken bezüglich der Durchsetzbarkeit eines theoretischen Entwurfs, den sie als zu elitär erachtet:

Der ärgere Schaden des Buches ist der, daß die Kritik der verschiedenen Richtungen in der modernen Literatur für uns einen Schlag ins Wasser bedeutet, weil sie sich nur auf französische Erscheinungen einläßt, welche wenig gekannt und daher von geringem Einfluß sind. Für uns sind die Evolutionen des Naturalismus noch nicht vollzogen; wir haben kaum die Anfänge einer psychologischen Dichtung; wie also totschlagen, was noch nicht geboren ist? ${ }^{43}$

Die Frage lautete also, wie es möglich war, dem Naturalismus den Prozess zu machen, wenn er doch eigentlich in Österreich noch gar nicht angekommen war. Tatsächlich lieferte jedoch genau diese Überlegung ein unumstößliches Argument, um die österreichische Literatur in einer neuen, europäischen Perspektive zu verankern. Denn in einem historischen Moment, in dem sich die kulturellen Triebkräfte auf das „Neue" zubewegten, eröffnete sich die einzigartige Möglichkeit, sich die neusten Erkenntnisse der neusten Literatur zunutze zu machen, ohne sich mit der Episode des Naturalismus länger aufhalten zu müssen.

Über den Naturalismus, aber auch über die Décadence und die spätere Begegnung mit der „Philosophie des Impressionismus“ hinaus, ${ }^{44} \mathrm{dem}$

43. Marie Herzfeld, „Hermann Bahr, ,Die Überwindung des Naturalismus“", Wiener Literatur-Zeitung, Jg. 2, Nr. 10, 15. August 1891, S. 10-11, hier S. 10.

44. Mit dem vitalistischen Reichtum der Wiener Episode des Impressionismus schließt bezeichnender Weise Bahrs Autobiographie, die 1923 unter dem Titel Selbstbildnis erschien, zu einem Zeitpunkt, als der Kritiker zunehmend an den Rand des literarischen und kulturellen Lebens rückte. So schreibt Bahr 1923 mit einem Anflug von Bitterkeit: „Es ist dreißig Jahre her, daß mich Harden den Mann von Übermorgen hieß. Ich bliebs lange. So lange, bis aus mir im stillen auf einmal ein Mann von vorgestern geworden war, der freilich, wenn ich mich recht besinn, doch 
Höhepunkt seiner produktiven schriftstellerischen Phase, erwies sich Bahr auch faktisch als Modernist, wobei die Vision des „Mannes von Übermorgen“, der seiner Zeit stets voraus ist, mit einem Projekt zur Deckung kommt, das den zeitgenössischen Erneuerungsgedanken auch auf die österreichische Literatur anwandte.

eigentlich immer schon insgeheim jenem Dränger über die Schultern sah: mein Zukunft mit Ungeduld verlangender Blick kehrt seit je doch am liebsten bei längst entschwundenen Vergangenheiten ein, da hole ich mir die Zukunft" (S. 2) 